\title{
FDTD Simulation of Radiation Characteristics of Half-Volume HEM- and TE-Mode Dielectric Resonator Antennas
}

\author{
Steven G. O’Keefe, Member, IEEE, Simon P. Kingsley, and Seppo Saario
}

\begin{abstract}
Cylindrical and rectangular dielectric resonator antennas (DRAs) using $\mathrm{HEM}_{11 \delta}, \mathrm{TE}_{11 \delta}$, and $\mathrm{TE}_{01 \delta}$ mode were examined to see the radiation pattern, impedance, field distribution, and resonant frequency that was achieved when the DRAs were bisected through an image plane by a conducting sheet. The resultant half DRAs are smaller in volume and have a more directional radiation pattern. The elevation angle of maximum radiation was lowered in some cases. Finite-difference time-domain simulation techniques were used.
\end{abstract}

Index Terms-Antennas, dielectric resonators, finite-difference time-domain (FDTD) methods.

\section{INTRODUCTION}

$\mathbf{T}$ HE small size, low cost, and low loss of the dielectric resonator antenna (DRA) has been well documented. In particular, those used above a conducting ground plane have been examined for impedance and radiation pattern [1], [2]. Further reductions in resonator dimensions have been demonstrated by utilizing the symmetry on certain resonant modes. The symmetry allows a reflecting metal plate or ground plane to bisect the resonator, thus halving the required volume [3], [4]. The radiation patterns of a cylindrical and rectangular DRA will be investigated in this paper. Similar dimensions to the structures described in [3] and [4] will be used for comparison. Results will be generated by use of computer simulations utilizing finite-difference time-domain (FDTD) techniques.

\section{Antenna Structure}

Two cylindrical DRA structures were examined. The first consisted of a dielectric cylinder placed over a ground plane. The cylinder has dimensions and permittivity as shown in Fig. 1. A probe was placed on a radius halfway to the outer edge the cylinder in such a position as to excite the $\mathrm{HEM}_{11 \delta}$ mode. This mode is supported by two horizontal contrarotating $\mathrm{H}$-field loops diametrically opposed. The probe is placed in the center of one of these. Because of the symmetry of the field lines in this mode, a conducting plane may be placed in the center of the cylinder, as also shown in Fig. 1, without affecting the resonant mode. The plane in this case does not extend past the edges of the dielectric. Note that the probe length was

Manuscript received June 6, 2001.

S. G. O'Keefe and S. Saario are with the Department of Microelectronic Engineering, Griffith University, QLD 4111 Nathan, Australia.

S. P. Kingsley is with the Department of Electronic and Electrical Engineering, University of Sheffield, S1 3JD Sheffield, U.K.

Publisher Item Identifier S 0018-926X(02)01707-6.

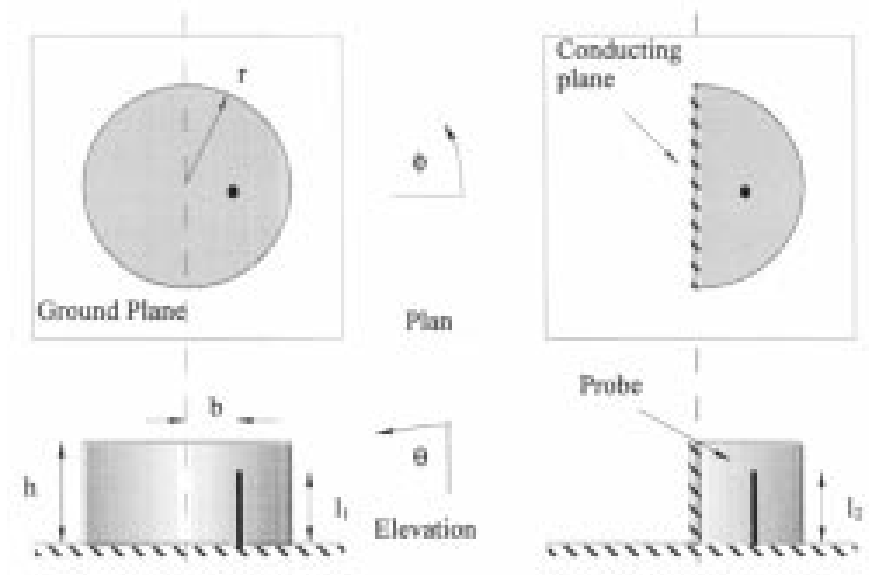

Fig. 1. Structure of the cylindrical and half-cylindrical HEM Hes $_{11}$ DRA. $\left(\varepsilon_{r}=\right.$ $12, r=36 \mathrm{~mm}, h=34 \mathrm{~mm}, b=18 \mathrm{~mm}, l_{1}=24 \mathrm{~mm}, l_{2}=20 \mathrm{~mm}$.)

changed in the half-cylinder. The second cylindrical structure is similar to that used in [4]. It consists of a cylindrical DRA in free space and its half-cylindrical equivalent bisected by a ground plane through a diameter of the cylinder. The structure and dimensions for this antenna are shown in Fig. 2. This DRA should resonate in the $\mathrm{TE}_{01 \delta}$ mode. Excitation was again by a probe. In the case of the half-cylinder, it was placed perpendicular to the ground plane as shown. The probe in the full cylindrical structure was a dipole probe placed symmetrically as shown in Fig. 2.

A similar approach is adopted for the rectangular DRA shown in Fig. 3. This DRA operates in the $\mathrm{TE}_{11 \delta}$ mode. Once again a coaxial probe was placed perpendicular to the ground plane at one end of the resonator. The probe position and length are altered in the half-DRA to maintain the correct impedance. Shortening the probe when near resonance generally reduces the real part of the impedance. The rectangular DRA is also $1 \mathrm{~mm}$ wider than that used in [3].

\section{FDTD MODEL}

Both the $\mathrm{HEM}_{11 \delta}$ mode cylindrical and $\mathrm{TE}_{11 \delta}$ mode rectangular DRAs were modeled using 1-mm cells in bounded and nongraded Cartesian simulation spaces of approximately $100 \times 100 \times 60$ cells. Berenger's perfectly matched layer (PML) absorbing boundary conditions (ABCs) were used to terminate the simulation space. The PML thickness was four cells with a parabolic conductivity profile. The residual reflection levels for this structure at the frequencies of interest were 


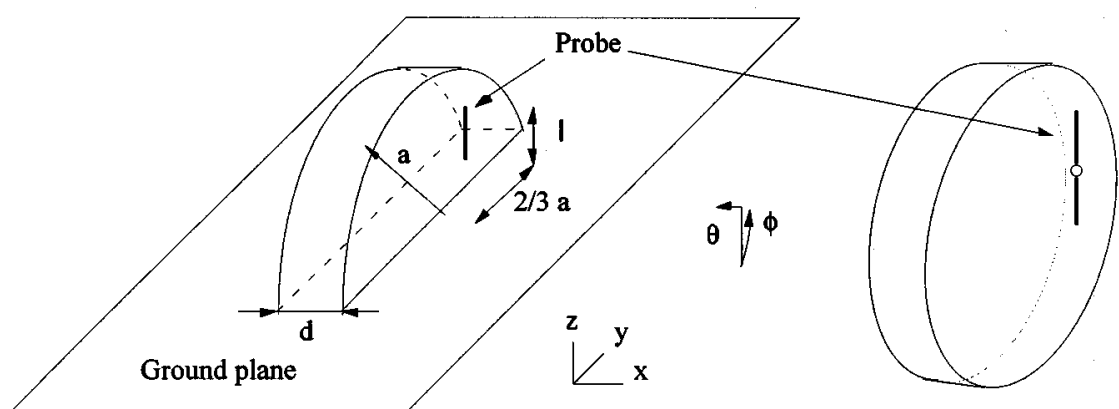

Fig. 2. Structure of the full and half-volume TE $E_{01}$ mode DRA. $\left(\varepsilon_{r}=12, a=7.4 \mathrm{~mm}, d=6.2 \mathrm{~mm}, l=3.2 \mathrm{~mm}\right.$. $)$

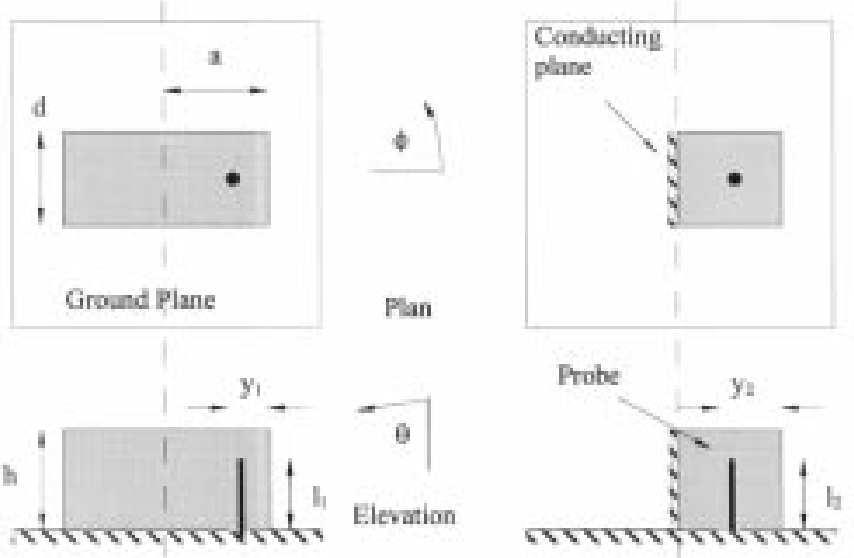

Fig. 3. Structure of the rectangular and half-rectangular $\mathrm{TE}_{11 \delta}$ mode DRA. $\left(\varepsilon_{r}=12, a=44 \mathrm{~mm}, h=40 \mathrm{~mm}, d=21 \mathrm{~mm}, l_{1}=22 \mathrm{~mm}, l_{2}=18 \mathrm{~mm}\right.$, $y_{1}=15 \mathrm{~mm}, y_{2}=22 \mathrm{~mm}$.)

approximately $-70 \mathrm{~dB}$. Since the split-field approach was used for the PML ABC, separate computational regions were used for efficient memory usage. The DRA structures were placed on an infinite perfect conducting ground plane and fed with gap excitation at the base of the probe. The $\mathrm{TE}_{01 \delta}$ mode cylindrical structure, being of much smaller dimensions, was simulated with a $0.2-\mathrm{mm}$ grid and similar boundary and ground-plane conditions. A Gaussian pulse of $40 \times 10^{-12} \mathrm{~s}$ was used for gap excitation to obtain the impedance and radiation pattern simulations, while sinusoidal gap excitation was used to obtain snapshots of the $\mathrm{E}$ - and $\mathrm{H}$-fields in the resonator.

An IBM SP2 supercomputer at the Queensland Parallel Supercomputing Facility was used for the computations with IBM RS/6000 wide nodes. Typical computation times were on the order of 20-30 h, running on a single node, due to the highly resonant behavior of DRAs. In all simulations, the dielectric was modeled as lossless.

\section{RESONANT FREQUENCY}

The resonant frequency of the full cylindrical $\mathrm{HEM}_{11 \delta}$ mode DRA was calculated with (1) described in [5]. This gave a frequency of $1.045 \mathrm{GHz}$. Note that this value is different to that calculated in [3], where an equation from [1] was used. As can be seen in [3], and from results in this paper, (1) yields a more accurate result

$$
f_{o}=\frac{18.972 \times 10^{8}}{2 \pi r \sqrt{\varepsilon_{r}+2}}\left[0.27+0.36 \frac{r}{2 h}+0.02\left(\frac{r}{2 h}\right)^{2}\right] .
$$
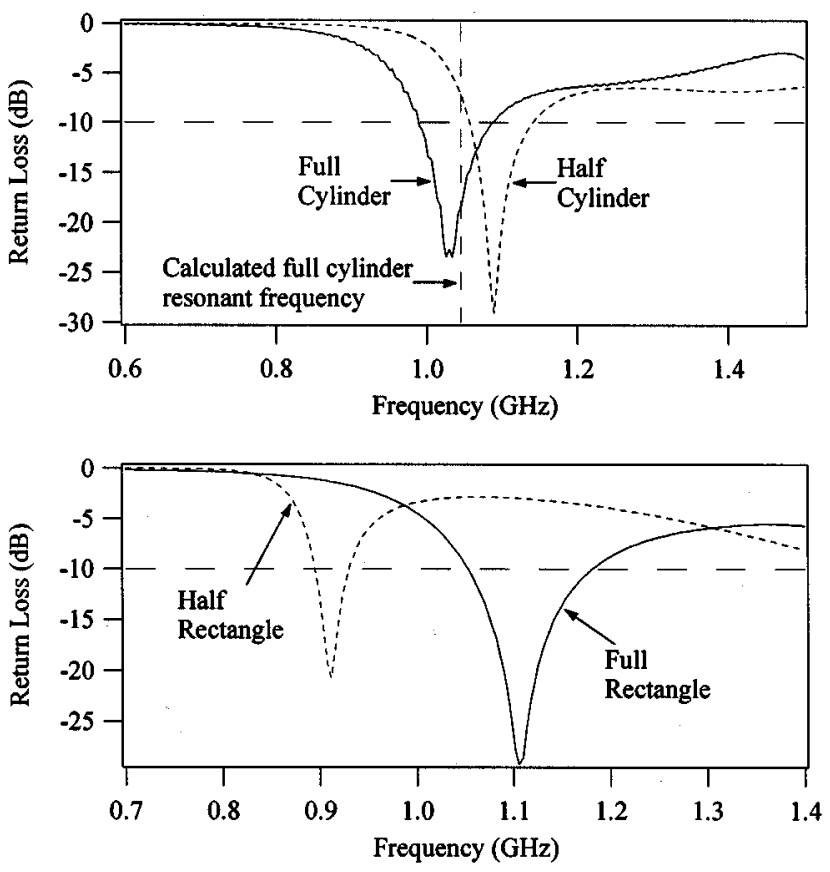

Fig. 4. Simulated return loss for the full and half $\mathrm{HEM}_{11 \delta}$ mode cylindrical and $\mathrm{TE}_{11 \delta}$ mode rectangular DRA.

The return loss of all DRAs was extracted from the FDTD simulation in order to ascertain the resonant frequency of each antenna. The return loss for the $\mathrm{HEM}_{11 \delta}$ mode cylindrical DRA and the $\mathrm{TE}_{11 \delta}$ mode rectangular DRA is shown in Fig. 4 for full and half structures. The error between calculated resonant frequency and minimum return loss frequency from the simulation for the full cylinder $\mathrm{HEM}_{11 \delta}$ mode DRA was found to be $15 \mathrm{MHz}$ or $1.4 \%$. Resonant frequencies for the full cylinder $\mathrm{HEM}_{11 \delta}$ mode and full rectangular $\mathrm{TE}_{11 \delta}$ mode were 1.03 and $1.105 \mathrm{GHz}$, respectively. The $-10 \mathrm{~dB}$ return loss bandwidths were $99 \mathrm{MHz}$ (9.6\%) for the cylinder and $127 \mathrm{MHz}(11.5 \%)$ for the rectangle. For the half-cylinder and rectangle, the resonant frequencies were $1.09 \mathrm{GHz}$ and $911 \mathrm{MHz}$, respectively, with bandwidths of $83 \mathrm{MHz}(7.6 \%)$ and $35 \mathrm{MHz}(3.8 \%)$. Note that the slightly lower frequencies observed for the rectangle compared to [3] are due to the slightly wider DRA. As expected, the resonant frequency of the half-DRAs had shifted. A finitedimensioned metal divider was not expected to fully mirror the fields, as some fields would be expected to spill around the ends of the divider. One might imagine that this would be more significant for the rectangle compared to the cylinder, as the divider 


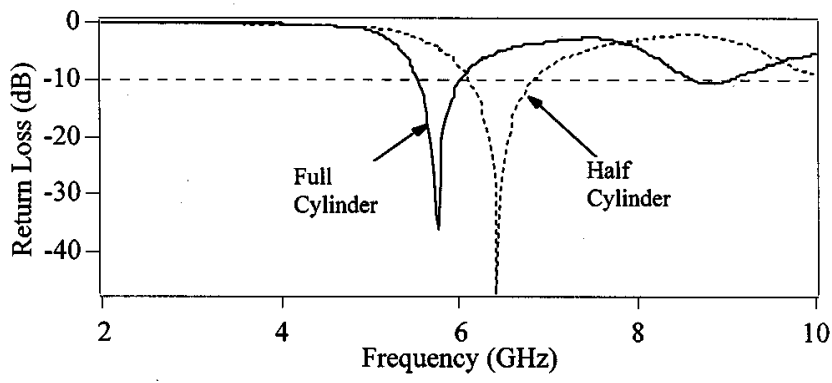

Fig. 5. Simulated return loss for the $\mathrm{TE}_{01} \delta$ mode full and half-cylindrical DRA.

ends are further from the areas of high field in the cylinder. The frequency shifts for cylinder and rectangle seem to indicate this.

The resonant frequency of the $\mathrm{TE}_{01} \delta$ mode cylindrical DRA was found to be $5.74 \mathrm{GHz}$ with a $-10 \mathrm{~dB}$ bandwidth of 475 $\mathrm{MHz}$, or $8.3 \%$, as shown in Fig. 5. This occurred with an overall probe length of $6.8 \mathrm{~mm}$. The input impedance of the antenna may be tuned by adjustment of the feed probe. The length of the probe was adjusted to give the best return loss, which corresponded to the zero crossing of the imaginary part of the impedance. In other words, the peak of the real part of the impedance was adjusted to $50 \Omega$. This is important for best excitation of the DRA. An incorrect probe length will give the best return loss at a frequency different from the DRA resonant frequency. Generally, when near resonance, shortening the probe will reduce the real part of the input impedance.

The half-cylinder $\mathrm{TE}_{01 \delta}$ mode DRA was then run with the ground plane in place. The optimum length for the probe was found to be $3.2 \mathrm{~mm}$, which differed from the value of $4.2 \mathrm{~mm}$ used in [4]. This was assumed to be due to the antenna in [4]'s having an air gap around and above the probe to allow adjustment of the probe. The effect of air gaps has been shown to cause difference between experimental and simulated results [6]. The resonant frequency, as shown in Fig. 5, was found to be 6.43 $\mathrm{GHz}$. This frequency is slightly higher than the value found in [4], where a $-10 \mathrm{~dB}$ return loss band of 5.8-6.2 $\mathrm{GHz}$ was obtained. This is thought to be due to the absence of any air gaps and the slight difference in resonator radius. Note that the $-10 \mathrm{~dB}$ return loss bandwidth is $730 \mathrm{MHz}$ or approximately $11.4 \%$. The resonant frequency of the half-cylinder DRA is significantly higher than the frequency of the full cylinder.

\section{FIELD Distributions}

By viewing the field distribution within the DRA, one first may understand the mode in which the DRA is resonating, and secondly may be able to make adjustments to the DRA shape, size, and probe position to maximize operation in the required mode. Being able to view these fields also helps in understanding the expected radiation pattern and how modification to the structure will influence the pattern. To examine only those fields due to one resonant mode within the DRA, the excitation must be at one frequency only.

The FDTD model was rerun with sinusoidal excitation fixed at the resonant frequency of each DRA as defined by the minimum return loss. A snapshot of the field intensity and direction

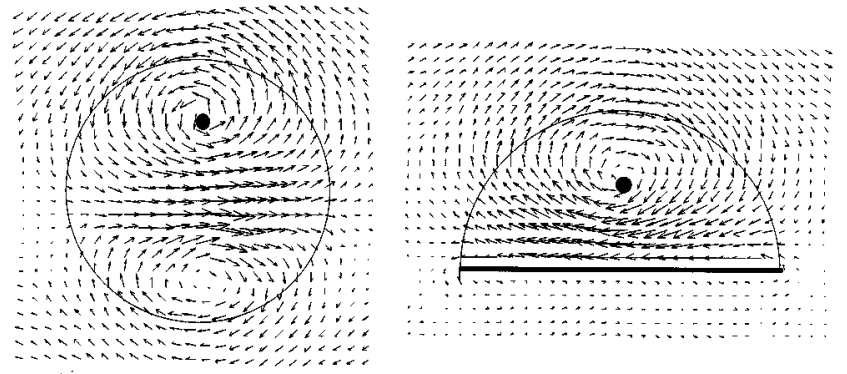

Fig. 6. H-field distribution for the $X Y$ plane in the full and half-cylindrical $\mathrm{HEM}_{11 \varepsilon}$ mode DRA $(Z=26 \mathrm{~mm})$.
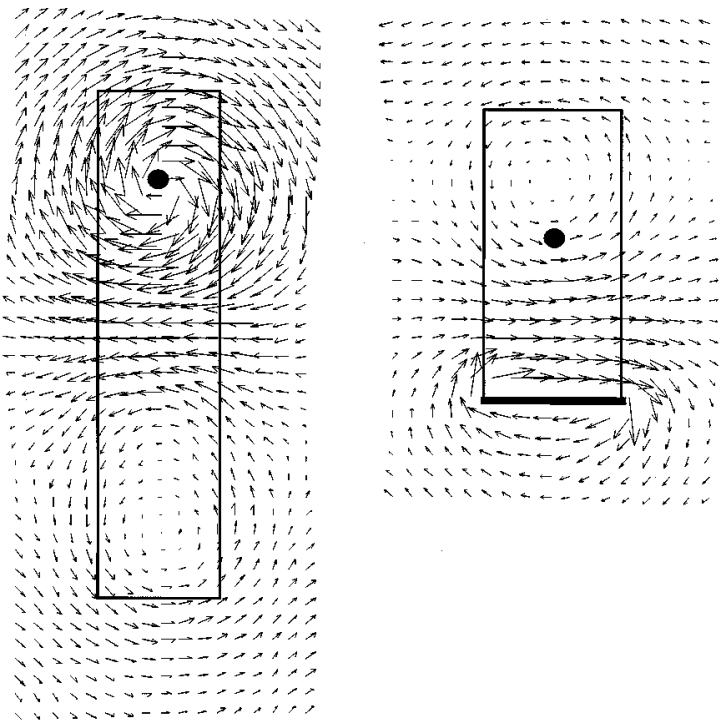

Fig. 7. H-field distribution for the $X Y$ plane in the full and half-rectangular $\mathrm{TE}_{11 \varepsilon}$ mode $\mathrm{DRA}(Z=26 \mathrm{~mm})$.

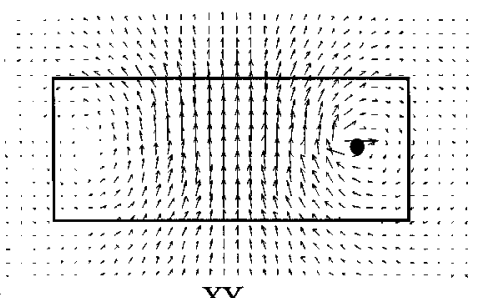

$\mathrm{XY}$

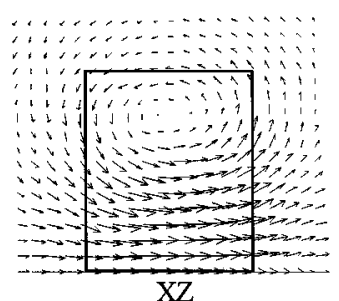

$\mathrm{XZ}$
Fig. 8. H-field distribution for the $X Y$ plane $(Z=2.8 \mathrm{~mm})$ and $X Z$ plane $(Y=$ center $)$ for the $\mathrm{TE}_{01 \varepsilon}$ mode cylindrical DRA.

was taken in a cut through each resonator. The cuts were generally positioned in the dielectric, but above the exciting probe. The H-fields only are displayed in Figs. 6-8. As expected, the divider in the half-cylindrical $\mathrm{HEM}_{11 \delta}$ mode DRA was quite effective in containing the field with only a little spillage around the end of the divider. The half-rectangular $\mathrm{TE}_{11 \delta}$ mode DRA, however, suffers badly. For certain parts of the excitation cycle, strong fields surround the metal divider. The slight asymmetry observed in the full rectangular structure may be due to the probe's being placed in a less than optimum position to excite this mode. Also, some field distortion is to be expected, as the natural field distribution may not be able to exist due to the 


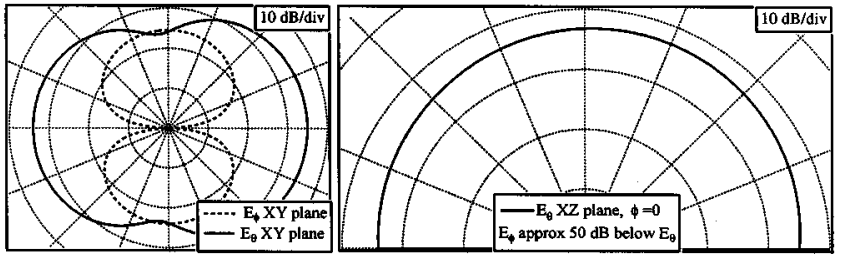

Full Cylinder

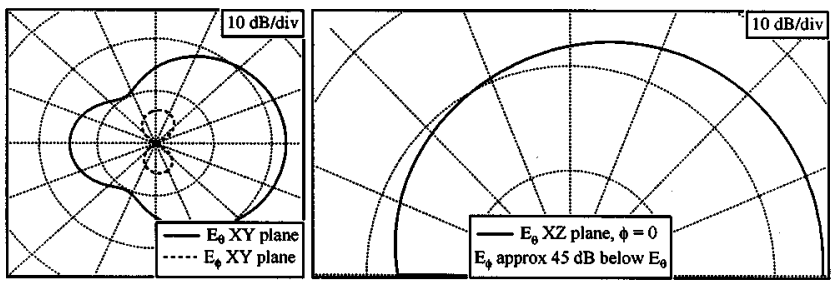

Half Cylinder

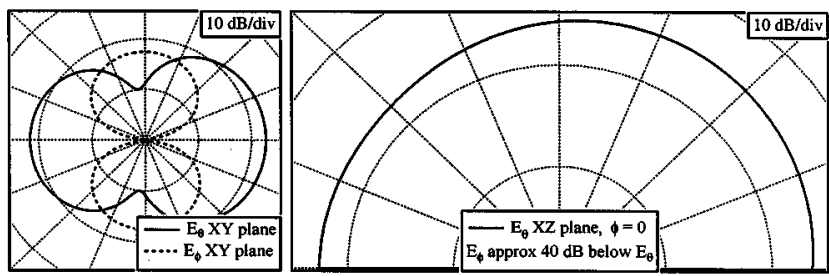

Full Rectangle
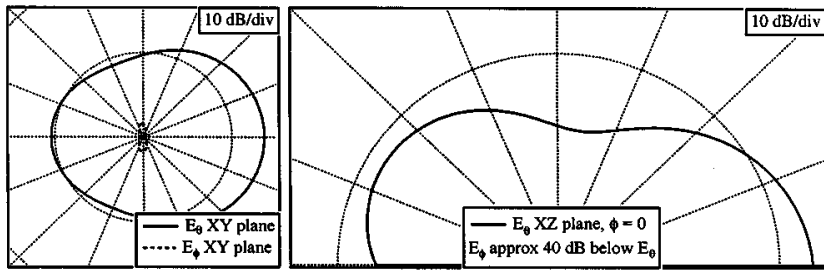

Half Rectangle

Fig. 9. Normalized $E_{\theta}$ and $E_{\phi}$ radiation patterns ( $X Y$ and $X Z$ planes) for full and half $\mathrm{HEM}_{11 \delta}$ mode cylindrical and $\mathrm{TE}_{11 \varepsilon}$ mode rectangular DRAs.

probe enforcing the field pattern at the excited pole. This effect is clearly evident in the half-rectangular field, taken above the probe, where the pole has moved off-center from the probe.The half-cylinder $\mathrm{TE}_{01 \delta}$ mode DRA H-field distribution is shown in Fig. 8. The two $X Y$ and $X Z$ cuts demonstrate how the cylinder can be divided by the ground plane without interfering with the fields for that mode. The infinite ground plane divides the DRA without any of the field spillage that occurred with the two previous examples.

\section{RADIATION PATTERNS}

The patterns for variations in $\theta$ ( $X Z$ plane) and $\phi(X Y$ plane) are plotted in Fig. 9 for the full and half $\mathrm{HEM}_{11 \delta}$ and $\mathrm{TE}_{11 \delta}$ mode DRAs at each antenna's respective resonant frequency. The radiation patterns for both full structures show the expected figure-eight pattern caused by the "magnetic dipoles." The probe can be thought of as a small segment from a vertically oriented current carrying loop. A magnetic field exists in the horizontal plane around this probe and also in the

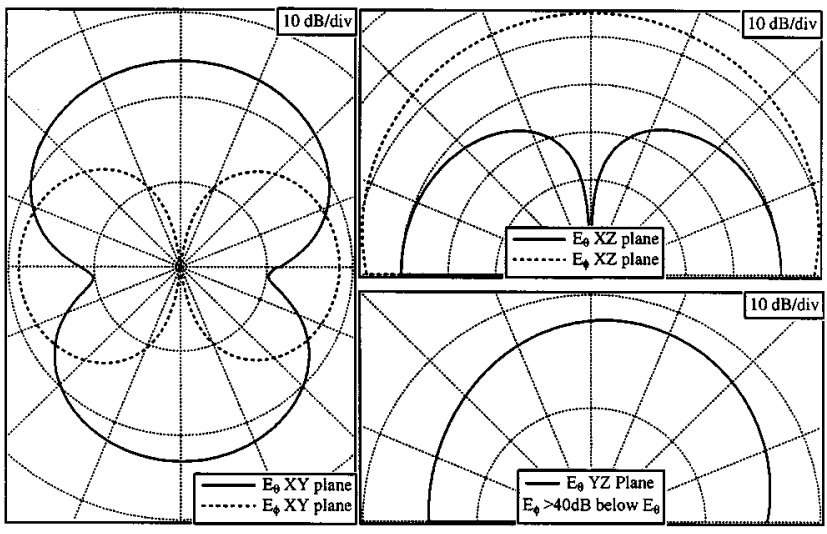

Fig. 10. Normalized radiation patterns in the $X Y, X Z$, and $Y Z$ planes for the $\mathrm{TE}_{01 \varepsilon}$ mode full- and half-cylindrical DRA.

opposite direction around the image of this probe, diametrically opposite in the DRA. This arrangement produces the fields that might be expected from two small diametrically opposite segments taken from a loop antenna, and is often called a magnetic dipole. This gives a similar radiation pattern, in azimuth, to a loop antenna. The patterns show improved directionality for the half-DRA in both cases. Also the elevation angle for maximum radiation has been reduced for both half-DRAs. The performance of the half-cylindrical structure is better than that of the half-rectangular structure in terms of usefulness as a directional antenna. The half-rectangular structure suffers from a poorer front-to-back ratio and an elevated rear lobe. It is thought that this inferior performance is due to the imperfect shielding provided by the metal divider.

Fig. 10 shows the radiation patterns for the $\mathrm{TE}_{01 \delta}$ mode halfcylindrical DRA. The pattern is half-toroidal and fairly symmetrical. The full cylindrical version pattern is not shown but was the expected figure-eight pattern in both $X Y$ and $X Z$ planes.

\section{CONCLUSION}

It can be seen that DRAs may be constructed using half of the volume of the normal structure, thus taking up half of the space and weighing nearly half as much. The resonant frequency of the half-DRA is altered slightly from that of a full DRA by between $6 \%$ and $17 \%$. The effect is most significant for the rectangular structure. It is observed that the shift in frequency is upwards for both the $\mathrm{HEM}_{11 \delta}$ and $\mathrm{TE}_{01 \delta}$ mode half-cylindrical DRAs but downwards for the $\mathrm{TE}_{11 \delta}$ mode half-rectangular DRA. The bandwidth of the DRAs also varies from full to half-symmetry. Both cylindrical DRAs experienced an increase in bandwidth when halved, while the rectangular DRA suffered a decrease in bandwidth.

The half-DRA also demonstrates a more directional radiation pattern than the full DRA. It is anticipated that directionality may also be further improved by extension of the conducting plane that divides the DRA. This should reduce the amplitude of the rear lobe. Both the $\mathrm{HEM}_{11 \delta}$ and $\mathrm{TE}_{11 \delta}$ mode half-DRAs and the $\mathrm{TE}_{01 \delta}$ mode DRA would seem suitable for satellite applications. However, the $\mathrm{HEM}_{11 \delta}$ and $\mathrm{TE}_{11 \delta}$ mode DRAs may find application for terrestrial antennas due to their lower radiation angles. 


\section{REFERENCES}

[1] S. A. Long, M. W. Mcallister, and L. C. Shen, "The resonant cylindrical dielectric cavity antenna," IEEE Trans. Antennas Propagat., vol. AP-31, no. 3, pp. 406-412, 1983 .

[2] A. A. Kishk, M. R. Zunoubi, and D. Kajfez, "A numerical study of a dielectric disk antenna above grounded dielectric substrate," IEEE Trans. Antennas Propagat., vol. 41, no. 6, pp. 813-821, 1993.

[3] M. T. K. Tam and R. D. Murch, "Half volume dielectric resonator antenna designs," Electron. Lett., vol. 33, no. 23, pp. 1914-1916, 1997.

[4] R. K. Mongia, "Half-split dielectric resonator placed on metallic plane for antenna applications," Electron. Lett., vol. 25, no. 7, pp. 462-464, 1989.

[5] R. K. Mongia and P. Bhartia, "Dielectric resonator antennas-A review and general design relations for resonant frequency and bandwidth," Int J. Microwave Millimeter Wave Comput.-Aided Eng., vol. 4, no. 3, pp. 230-247, 1994

[6] S. Shum and K. Luk, "FDTD analysis of probe-fed cylindrical dielectric resonator antenna," IEEE Trans. Antennas Propagat., vol. 46, no. 3, pp. 325-333, 1998

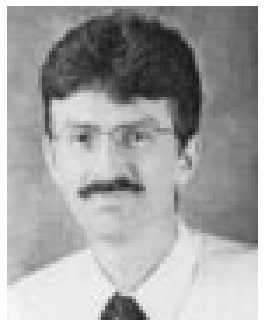

Steven G. O'Keefe (S'85-M'91) was born in Brisbane, Australia. He received the B.Sc. degree in physics, mathematics, and electronics and the Ph.D. degree in geophysical electromagnetics from Griffith University, Australia, in 1985 and 1996, respectively, and the B.Sc. degree (Hons.) in electronics and the M.Sc. degree in biomedical electronics from LaTrobe University, Australia, in 1986 and 1990, respectively.

$\mathrm{He}$ is currently a Senior Lecturer in the School of Microelectronic Engineering, Griffith University, and is a Consultant for Antenova, Ltd. U.K. His research interests are in the areas of compact mobile antenna design and electromagnetic geophysics.

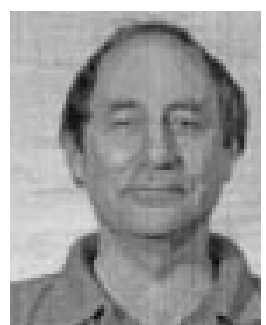

Simon P. Kingsley received the B.Sc. degree in physics from Nottingham University, U.K., the M.Sc. degree in radio astronomy from Jodrell Bank, Manchester University, U.K., and the Ph.D. degree in space physics from Sheffield University, U.K.

In 1975, he was a Research Fellow at the Institute of Atmospheric Physics, Bologna, Italy, developing new techniques for studying the upper atmosphere by radar. After several years as an Advanced Research Fellow at Sheffield University, he joined the GEC-Marconi Research Centre to carry out research into over-the-horizon radar. In 1987, he rejoined Sheffield University as a Lecturer and then Reader. In 2001, he became Chief Scientist, Antenova, Ltd., U.K. In 1997, he was a Faraday Lecturer for the Institution of Electrical Engineers, U.K. In 1999, he was the Sir Alan Sewell Visiting Fellow at Griffith University, Brisbane.

Dr. Kingsley is a Fellow of the Royal Astronomical Society and the Institution of Electrical Engineers.

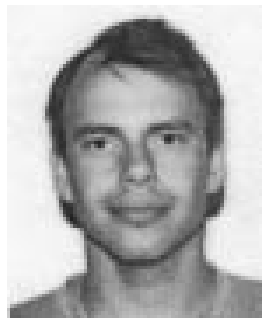

Seppo Saario was born in Pori, Finland, in December 1973. He received the B.E. degree (with honors) in microelectronic engineering from Griffith University, Australia, in 1995, where he is currently pursuing the Ph.D. degree.

During his undergraduate and postgraduate studies, he spent two years as an exchange student at the University of Electro-Communications in Japan. He currently is engaged in the numerical analysis of various electromagnetic problems, including small directional antennas for mobile communications. His research interests include time-domain numerical methods, high-performance parallel computing, mobile communications, directional antennas, and millimeter-wave systems. 